Pour une politique de rapprochement entre les peuples autochtones et les nouveaux arrivants francophones à Edmonton (Alberta)

\author{
Nathalie Kermoal \\ Faculty of Native Studies, University of Alberta \\ Paulin Mulatris \\ Campus Saint-Jean, University of Alberta
}

aboriginal policy studies Vol. 3, no. 1\&2, 2014, pp. 135-148

This article can be found at:

http://ejournals.library.ualberta.ca/index.php/aps/article/view/20923

ISSN: 1923-3299

Article DOI: http://dx.doi.org/10.5663/aps.v3i1-2.20923

aboriginal policy studies is an online, peer-reviewed and multidisciplinary journal that publishes original, scholarly, and policy-relevant research on issues relevant to Métis, non-status Indians and urban Aboriginal people in Canada. For more information, please contact us at apsjournal@ualberta.ca or visit our website at www.ualberta.ca/nativestudies/aps/.

UNIVERSITY OF ALBERTA

FACULTY OF NATIVE STUDIES
Aboriginal Affairs and Aboriginal Affairs and
Northern Development Canada

Affaires autochtones et Développement du Nord Canada 


\title{
Pour une politique de rapprochement entre les peuples autochtones et les nouveaux arrivants francophones à Edmonton (Alberta)
}

\author{
Nathalie Kermoal \\ Faculty of Native Studies, University of Alberta \\ Paulin Mulatris ${ }^{1}$ \\ Campus Saint-Jean, University of Alberta
}

Résumé: S’appuyant sur des données qualitatives recueillies à Edmonton entre avril 2011 et mars 2012 auprès de participants autochtones et d'immigrants africains francophones à Edmonton, cet article cherche à comprendre comment les nouveaux immigrants francophones interagissent avec les peuples autochtones, deux groupes perçus par la société dominante comme des groupes marginalisés. Les résultats de notre étude exploratoire montrent un tableau contrasté des perceptions que les uns ont des autres. Bien que l'étude révèle des malentendus et des incompréhensions de part et d'autre, les participants reconnaissent l'importance de développer des solidarités, afin de faciliter l'ouverture et le dialogue dans une grande ville comme Edmonton. Pour les participants, la construction de ces ponts mènerait vers un Canada nouveau et plus inclusif.

En 2010, dans un article intitulé "Canadian Multiculturalism: Global Anxieties and Local Debates ", Banting et Kymlicka insistaient sur l'importance d'examiner le multiculturalisme à travers les liens que développent les Autochtones et les immigrants dans les villes de l'Ouest étant donné leur poids démographique et le partage de certains espaces publics. Selon ces auteurs, "while constitutionally speaking they may fall under different laws and regulations, the practical reality is that they often share public services and public space [...] More work needs to be done to explain how they interact » $(2010,64)$. Banting et Kymlicka présument de l'existence de relations; or, la réalité est plus complexe, du fait du poids de l'Histoire et des rapports de pouvoir colonialistes que la rhétorique du multiculturalisme ne peut effacer.

Notre article reprend les éléments d'une recherche réalisée entre avril 2011 et mars 2012, menée auprès de participants autochtones et d'immigrants africains francophones d'Edmonton. Lobjectif premier était de déterminer ce que les uns pensent des autres et de comprendre ce qui peut empêcher la rencontre entre les peuples dans un espace urbain. Lobjectif pratique de la recherche était, au-delà des cloisonnements institués par

1 Les auteurs aimeraient remercier les évaluateurs anonymes de cet article pour leurs commentaires constructifs. Cette recherche a été possible grâce à une subvention du CRSH par l'entremise du réseau DIALOG : le réseau de recherche et de connaissances relatives aux peuples autochtones, et grâce à une subvention du fonds EFF-SAS, University of Alberta.

aboriginal policy studies, vol. 3, no. 1\&2, 2014

135

www.ualberta.ca/nativestudies/aps/

ISSN: 1923-3299 
l'État (bilinguisme, multiculturalisme, affaires autochtones, etc.) ou par les communautés (ghettoïsation, enclaves ethniques, etc.), de contribuer au développement d'initiatives de sensibilisation et de rapprochement entre les populations concernées aussi bien au niveau communautaire qu'universitaire. Nous espérions ainsi rassembler et analyser les aspects pouvant servir de base au développement de solidarités et faciliter l'esprit d'ouverture, le dialogue entre les populations autochtones et les immigrants francophones d'origine africaine.

Parmi les raisons qui justifient cette étude, le poids démographique croissant de ces populations dans le tissu social et le tissu urbain canadien, l'importance et le parallélisme des enjeux sociaux qui les concernent nous ont paru déterminants. En effet, depuis une dizaine d'années, les populations autochtones et immigrantes ont connu des changements qui ont largement contribué à la croissance démographique du Canada des dernières années. En 2006, Statistique Canada établissait que plus d'un million de personnes se sont identifiées comme Autochtones (Inuits, Métis, Premières nations). En 2011, les Premières nations, les Métis et les Inuits comptaient pour 4,3\% de la population totale du Canada (Statistique Canada 2013a). Cette population autochtone est de plus en plus urbanisée, alors que 54 \% d'entre eux vivent en ville (Statistique Canada 2013a). En outre, c'est une population jeune : les enfants et les jeunes de 24 ans et moins forment près de la moitié $(46,2 \%)$ de la population autochtone, comparativement à $27,1 \%$ pour la population non autochtone (Statistique Canada 2013a). En 2011, l'âge médian de la population autochtone était de 28 ans, comparativement à 41 ans pour la population non autochtone (Statistique Canada, 2013a).

Les vagues successives d'immigration ont aussi transformé le paysage urbain du Canada, confirmant le caractère fortement diversifié de ce qu’on appelle communément la « mosaïque ethnoculturelle canadienne ». L'augmentation de l'immigration africaine a été une des causes importantes de la diversification de la population canadienne. Environ 145700 immigrants africains ont été accueillis entre 2006 et 2011 (Statistique Canada 2013b). Comme on l'observe dans le dernier recensement de 2011, ceci a sans doute contribué à augmenter le poids démographique des minorités visibles, soit 19,1\% de la population totale du Canada, comparativement à 16,2 \% en 2006 (Statistique Canada 2013b). Les Noirs, qui forment désormais le troisième groupe en importance parmi ces immigrants, représentent 15,1 \% (945 700 personnes) de cette population croissante (Statistique Canada 2013b). Avec un âge médian de 29,4 ans, cette nouvelle population d'origine africaine a la particularité d'être relativement jeune (Statistique Canada 2013b). À l'instar des Autochtones, ils font en général partie de la catégorie de la population considérée comme «à risque ». Ils ont, pour la plupart, été victimes du colonialisme, de la persécution, de la guerre, de la violence ou de traumatismes dans leur pays d'origine, et évoluent dans un environnement économique très fragile au Canada (Thurston, McGrath et Sehgal 1993; Lévesque 2005). Mais ces parallélismes suffisent-ils vraiment pour définir un terrain de rencontre entre ces populations? En d'autres termes, peut-on considérer ces faits sociaux et historiques comme une occasion de cet apprentissage interculturel qui, selon Giust- 
Desprairies et Müller (1997, 1), « se produit lorsque surgissent des difficultés, des processus de différenciation, des antagonismes qui ne peuvent être perçus que progressivement et qui ne peuvent être surmontés qu'ensemble »? Pourtant, même si ces populations immigrantes francophones africaines sont censées transporter des vécus coloniaux douloureux, ceux-ci sont - vu leur âge - quelques fois lointains, et courent le risque de s'effriter au cours de leurs longues années de réinstallation au Canada marquées par le discours de l'intégration. Aussi est-il important de se demander si, malgré la similarité de ces vécus mémoriels, il existe chez ces populations immigrantes une bonne connaissance des réalités historiques et des revendications des peuples autochtones du Canada. Dans quelle mesure, par exemple, le discours social de la société dominante appris par les nouveaux arrivants (jeunes et adultes) contribue-t-il à transmettre des stéréotypes qui véhiculent une image plutôt négative de l'Autochtone?

Lensemble de ces interrogations nous amène, dans un premier temps, à examiner la place des droits autochtones dans une rhétorique de politique multiculturelle afin de mieux situer la pertinence de notre contribution. Ensuite, nous exposerons la méthodologie retenue pour mener à bien notre étude. Enfin, dans une troisième partie, nous présenterons une analyse préliminaire des résultats de notre enquête auprès d'un groupe de participants autochtones et africains à Edmonton.

\section{Les droits des Autochtones et la politique multiculturelle du Canada}

Le Canada arbore fièrement sa politique multiculturelle et il fait figure de modèle en la matière. Cette politique est perçue par des intellectuels comme John Ralston Saul comme profondément autochtone, parce que fondée sur un équilibre entre les droits individuels et collectifs (Saul 2008, 62-63). Quoique les Autochtones ne réfutent pas l'idée du multiculturalisme, ils sont cependant sceptiques face à la rhétorique qui l'entoure. Selon eux, cette dernière aurait tendance à minimiser leur rôle dans la société canadienne. En effet, peu de temps après l'officialisation en 1971 de la politique multiculturelle au Canada, des leaders autochtones la dénonçait, comme Harry Daniels en 1979, parce qu'ils considéraient qu'elle nourrissait « le mythe des deux peuples fondateurs en y mélangeant des oufs de Pâques ukrainiens, des raisins italiens, ou de la banique métisse pour un peu de saveur supplémentaire " (Daniels 1979, 51)2. Les Autochtones récusent, entre autres, l'étiquette de "groupes ethniques » que certains leur attribuent et déplorent la « folklorisation » de leurs cultures (Daniels 1979). Également, ils s'inquiètent des conséquences de la méconnaissance de l'histoire de ce pays par les nouveaux arrivants sur l'avancement de leurs revendications territoriales ou politiques (Daniels 1979; Lawrence 2011). Selon Sunera Thobani, en mettant l'accent sur la tolérance et la diversité, la politique multiculturelle aurait tendance à discréditer les revendications des Autochtones, notamment leur statut spécial de premiers occupants du territoire: "Aboriginality is instead devalued as only one

2 « The government continues to feed us the 2 founding nations myth while tossing in some Ukrainian Easter eggs, Italian grapes, or Métis bannock for some extra flavor» (Daniels 1979, 51). Traduction libre par Nathalie Kermoal. 
among several cultures that needs to be harnessed for the cultural enrichment of nationals. Multiculturalism has demanded that Aboriginals extend their tolerance to the presence and claims of these other cultures " (Thobani 2007, 174). Pour l'auteure, l'identité nationale canadienne est profondément ancrée dans un passé colonial violent ainsi que dans des notions qui mettent de l'avant la suprématie raciale blanche et la supériorité civilisationnelle occidentale (Thobani 2007, 249).

L'effacement de l'histoire autochtone ainsi que l'idée de territoire sans maitre ou Terra Nullius sont encore très présents au Canada malgré les avancées des droits des Autochtones depuis les années 1970 (voir Flanagan 2000). En effet, pour la politologue autochtone Joyce Green, l'histoire du Canada ressemble à un palimpseste, car

les nouveaux arrivants qui sont venus occuper le territoire ont superposé leur expérience et leur vécu sur le parchemin national, estompant du coup, voire niant les traces laissées par les peuples autochtones. Mais le cumul des réécritures successives fait le palimpseste et le définit dans sa totalité; les anciennes strates modulent, façonnent même, en quelque sorte, les ajouts subséquents. À l'heure où l'État canadien cherche, dit-on, à redéfinir sa relation avec les peuples autochtones et à aménager les voies de la réconciliation, le vrai défi consiste précisément à ne pas perdre de vue l’empreinte autochtone et le sort qui lui a été réservé. (Green 2004, 12)

De surcroît, l'anthropologue Ghassan Hage a souligné à propos de l'Australie qu'il était complexe pour un immigrant de participer pleinement au paysage politique du pays d'accueil quand ce dernier a été pollué par le colonialisme et ses suites et que la grande majorité des citoyens vivent une amnésie collective (Hage 2001). Au Canada, comme en Australie, les réécritures multiples d'appartenance et d'exil sont à la fois enchevêtrées, interdépendantes, souvent concordantes et très fréquemment irréconciliables (Offord 2006, 13). Les Autochtones et les immigrants sont alors obligés de négocier les réalités multiples du paysage canadien.

Même si les pratiques du colonialisme ont changé avec le temps et que le colonisateur n'a plus tout à fait le visage qu'il avait au XIX ${ }^{\mathrm{e}}$ siècle, les rapports de pouvoirs perdurent. Malgré la volonté de la part de l'État canadien de présenter le multiculturalisme comme la voie qui tend vers l'unité, les Autochtones, eux, perçoivent cette politique comme la voie qui tend vers l'uniformité. L'espace national canadien devient à leurs yeux un rouleau compresseur homogénéisant. Ils établissent une corrélation entre la politique d'assimilation mise de l'avant par le gouvernement fédéral par l'entremise du livre blanc de 1969 et la création de la politique du multiculturalisme en 1971 (Lawrence 2011, 254), ces deux politiques ayant pour but d'affaiblir leur quête d'autodétermination. Selon la sociologue Himani Bannerji (2000, 87-124 in Salée 2005,67), le multiculturalisme "gomme l'expérience coloniale et le caractère profondément eurocentrique de l'espace national canadien dans le but de recomposer et de relégitimer la nation afin de la rendre attrayante au plus grand nombre ». L'État canadien laisse donc miroiter un idéal qui tend vers l'ouverture à l'Autre (Autochtone, immigrant, etc.) et la mise en place d'un espace politique accommodant (Salée 2005). Ainsi, au Canada, le discours et les politiques sur la citoyenneté, le multiculturalisme et 
l'immigration assurent « la pérennité d'une certaine vision de la société canadienne, des rapports sociaux qui la marquent et des vecteurs culturels qui la traversent » (Labelle et Salée 1999, 140). L'idéal multiculturel canadien empêche de rendre la quête déterministe des peuples autochtones visibles aux yeux des nouveaux arrivants, ces derniers se percevant externes au projet colonialiste canadien. Il est certain que la relation que les nouveaux arrivants entretiennent avec l'État canadien est complexe puisqu'ils sont, à l'instar des Autochtones, marginalisés. Toutefois, cette posture menant vers l'indifférence et la reproduction de stéréotypes perpétués par la société dominante ne favorise pas le dialogue ni une meilleure connaissance des cultures autochtones et de leurs revendications.

Afin de contourner l'incompréhension mutuelle, il est impératif d'encourager les gens de différentes cultures à apprendre les uns des autres. D’ailleurs, un projet de recherche mis de l'avant par le gouvernement canadien en 2006 intitulé Comprendre la réalité « 3 $M » d u$ Canada au XXI siècle notait qu'il existe un décalage important entre les politiques de multiculturalisme et la réalité sur le terrain (Gouvernement du Canada 2009). Comme nous l'avons souligné dans notre introduction, la diversité culturelle, telle que nous la connaissons aujourd'hui, se manifeste principalement en milieu urbain. C'est en ville que la grande majorité des Autochtones et des immigrants vivent et que les groupes interagissent et se côtoient. David Newhouse et Evelyn Peters soulignent d'ailleurs que :

cet aspect a été négligé par la Commission royale sur les peuples autochtones (1996), sans toutefois que les commissaires manquent d'en évaluer l'importance. Il ne s'agit pas d'un problème simple; toutefois, l'augmentation de la population urbaine contraint à dégager et à mettre en évidence des stratégies qui intègrent les communautés autochtones dans la vie économique et sociale urbaine en mettant en valeur leur contribution culturelle, sans oublier leur contribution juridique particulière. $(2003,311)$

Cependant, malgré ces nouvelles réalités et une meilleure compréhension du phénomène d'urbanisation des Autochtones (Newhouse and Peters 2003), très peu d'études existent sur les relations qu'entretiennent les peuples autochtones vivant dans les grandes villes canadiennes avec les communautés immigrantes. Lucía Madariaga-Vignudo (2009) s'est penchée sur le centre-ville de Winnipeg pour étudier les perceptions qu'avaient les Autochtones des réfugiés africains. Les résultats de son enquête révèlent un tableau plutôt contrasté. Si certains ont une perception négative des réfugiés, causée en partie par la concurrence pour les emplois et les logements subventionnés, d'autres semblent avoir un point de vue plus favorable, car ils peuvent s'identifier aux nouveaux arrivants en tant que minorités racisées. Toutefois, son étude ne révèle pas le point de vue qu'ont les réfugiés des Autochtones. En outre, les municipalités souhaitent aussi renforcer les liens entre Autochtones et communautés d'immigrants. Par exemple, en 2010, la Ville de Vancouver lançait un projet intitulé Dialogues, impliquant les Premières nations, les communautés urbaines autochtones et les immigrants de la ville. Vingt-sept partenaires communautaires et plus de deux mille personnes y ont participé (Ville de Vancouver 2011). La phase I a surtout mis l'accent sur les cercles de dialogue, la recherche communautaire, les visites d'échanges 
culturels, un programme pour les jeunes et les aînés ainsi qu'un projet patrimonial de quartiers (Ville de Vancouver 2011). Le projet est actuellement dans sa deuxième phase, celle de la mise en œuvre des idées proposées par les collectivités participantes (Ville de Vancouver 2011).

L'examen en profondeur des relations entre Autochtones et non autochtones dans les régions urbaines s'avère donc primordial puisque la forte croissance démographique d'un côté comme de l'autre a transformé les collectivités, les quartiers, les écoles, les lieux de travail et les institutions sociales (Gouvernement du Canada 2009; Banting et Kymlicka 2010). Mais qu'en est-il exactement des relations entre les Autochtones et les immigrants francophones africains vivant en Alberta, une province majoritairement anglophone?

\section{Approche méthodologique}

$\mathrm{Du}$ fait de son profil démographique, Edmonton s'avère une ville idéale pour entreprendre une étude sur le dialogue entre les populations autochtones et les immigrants francophones d'origine africaine. La population totale d'Edmonton en 2011 était de 1159869 habitants (Statistique Canada 2013c). Edmonton se classe au deuxième rang des villes (après Winnipeg) ayant une plus forte population autochtone, avec sa population florissante de 61765 autochtones (Statistique Canada 2013c). Cette croissance concerne aussi la population immigrante : les statistiques de 2011 indiquent que l'Alberta (après l'Ontario, le Québec et la Colombie-Britannique) est le quatrième lieu de destination des nouveaux arrivants parmi lesquels on compte de plus en plus d'Africains (Chagnon 2013, 9).

Déjà selon le recensement de 2006, les immigrants représentaient 17 \% (554 130) de la population albertaine. Parmi ceux-ci, on compte 5,4\% (29923) d'immigrants francophones dont $24 \%$ sont d’origine africaine (Statistique Canada 2006). Ceux qui vivent à Edmonton sont originaires de la République démocratique du Congo, du Rwanda, de l'Algérie, du Maroc et du Burundi (Statistique Canada 2006). Comme les Autochtones, ces immigrants travaillent depuis plusieurs années à la mise en place d'institutions communautaires et ont déjà plusieurs associations à leur actif.

Pour mener notre recherche, nous avons adopté une approche ethnosociologique qui s'insère dans un courant méthodologique qualitatif, interprétatif et comparatif. Une telle approche, recommandée par Sanséau $(2005,34)$, nous a permis d'accéder au vécu des participants, dans la singularité de leurs expériences, tout en tenant compte à la fois des moments critiques survenus dans leurs interactions avec les autres et des contextes de vie à travers lesquels ils naviguent. Évoquer les notions d' « interactions » et de " contextes de vie » nous a amenés à prendre en considération ce que Longhi $(2010,227)$ nomme « formations discursives ", c'est-à-dire les conditions de production des discours (textes) qui font que tout discours (texte) renvoie toujours au vécu, aux rapports sociaux; il est toujours en rapport avec d'autres discours qui en forment la trame et même, peut-être, la clé de lecture. Cette situation d'intertextualité (interdiscours) interdit toute forme de clôture symbolique. Elle est le lieu privilégié d'une émergence du sens de soi, des autres et du monde, c'est-à-dire le 
lieu d'une construction identitaire dans la mesure où — comme le suggèrent Dubar (2000), Maingeneau (1994) et Sébastien (2006) - cette situation s'entend comme un processus complexe sétablissant par/avec/contre les autres, une tension constante entre l'individuel et le collectif, le soi et l'autrui. L'intégration de cette dimension dialogique des identités dans cette étude nous a amenés à nous demander comment différents discours - produits par la société dominante de souche européenne, les milieux autochtones ou, plus récemment, par les nouveaux arrivant africains - interfèrent dans le processus de construction/ reconstruction identitaire des participants autochtones et africains.

Comme chercheurs, et comme le suggère l'ethnographie critique, nous avons dû adopter une démarche critique pour accéder aux discours, aux sous-discours et aux vécus qui les sous-tendent. Il nous a fallu ainsi remettre en question nos propres jugements, les concepts, les catégories ou les découpages préétablis, pour nous positionner dans une situation d'interaction afin de découvrir les mécanismes sous-jacents, d'associer la théorie à l'expérience et d'accroitre la validité de notre étude (Farmer et Labrie 2008, 380). Cette implication personnelle nous a aussi obligés à tenir compte des enjeux d'équité et de justice sociale (Denzin et Lincoln 2005), des enjeux linguistiques auxquels, d'une façon ou d'une autre, les participants font quotidiennement face. En effet, notre étude, soulignons-le, concerne des groupes d'individus que la littérature sociologique canadienne a tendance à considérer comme victimes de plusieurs formes d'inégalités sociales (Campagne 2000 2012; Gilmore 2006).

Notre objectif premier était de documenter les perspectives des participants et d'essayer de dégager les thématiques émergentes dont la compréhension est un préalable à létablissement des ponts entre les communautés autochtones et les nouvelles communautés immigrantes francophones africaines. Nous avons organisé quatre entretiens de groupe d'une durée maximum d'une heure chacun. Vingt-huit répondants, dont onze immigrants et dix-sept Autochtones, répartis en deux groupes homogènes, ont participé à ces discussions. Cette répartition nous a paru pertinente parce que non seulement nous abordions un sujet délicat, mais aussi parce que nous tentions de faire rencontrer dans un contexte d'études des populations qui, bien qu'ayant des parcours historiques supposément similaires (colonisation), n'avaient pour la plupart pas encore eu l'occasion de se côtoyer ou de se parler dans leurs vies quotidiennes. Les discussions en groupe permettaient ainsi de délier les langues dans un cadre homogène sans que les participants ne se sentent personnellement compromis dans un contexte où la partie " adverse " serait en présence. Cette distinction entre les deux groupes se justifiait aussi parce que nous pensions qu'une approche comparative permettrait d'isoler à l'intérieur d'un même cas les aspects relevant des différences culturelles. Le choix du français et de l'anglais comme langues d'entrevue, respectivement avec les participants africains et autochtones, était un premier indice de cette différence. Lobjectif était, pour nous, de permettre à chacun de mieux exprimer ses vécus dans la langue la plus maitrisée, en même temps que samorceraient les possibilités de rencontre au-delà de ce premier écueil linguistique; car au fil des discussions, la volonté de mieux se faire comprendre a parfois poussé quelques participants à choisir la langue la 
mieux comprise par l'autre pour mieux lui exprimer sa pensée (lors des rencontres mixtes subséquentes).

Pour chacun des deux groupes, deux entretiens ouverts rassemblant les mêmes participants étaient organisés. Le guide d'entretien comportait quelques thèmes de base, dont les vécus historiques, la connaissance de l'autre, le dialogue, les expériences partagées, les perspectives d'avenir, les possibilités de rencontre sur fond des vécus historiques ou culturels comparables, etc. Ces quelques thèmes ont servi de point de départ pour des discussions qui, bien souvent, débordaient des considérations initiales. Entre les deux entretiens respectifs, nous avons joué le rôle de médiateurs qui rapportaient les perspectives venant de l'autre groupe afin d'alimenter la discussion et de préciser les aspects en discussion de façon évolutive. Les discussions suivantes revenaient parfois sur les mêmes thèmes, mais en creusant davantage les aspects moins abordés au cours des discussions précédentes. Cette phase initiale était suivie par des discussions mixtes durant lesquelles les perspectives émergeant de la phase précédente étaient validées/invalidées dans un cadre plus ouvert et diversifié, avec des participants venant des différents groupes autochtones et immigrants.

Comme quelques études postcoloniales et féministes le suggèrent, les perspectives par rapport aux vécus sociohistoriques sont multifactorielles (Davis 2007). Ainsi, la prise en compte de l'intersectionnalité des concepts de race, de genre, d'âge ou de groupes/classes d'appartenance nous a semblé une meilleure approche pour viser à la fois la complexité du phénomène étudié et le niveau de saturation de l'information recherché dans la collecte des données. Il nous a paru ainsi nécessaire d'intégrer plusieurs caractéristiques dans la sélection des participants afin de répondre au caractère complexe du phénomène en étude. En effet, outre l'appartenance au groupe, la sélection des participants a été faite en fonction de deux autres critères, le genre et le groupe d'âge (18-45ans ou 45 ans et plus). Ces deux critères supplémentaires nous ont semblé importants parce que l'âge est un facteur pour la connaissance des vécus sociohistoriques propres aux participants. Les plus jeunes semblent être, par exemple, moins informés sur l'histoire coloniale expérimentée par leurs aînés. De plus, la perspective par rapport aux thèmes analysés peut varier suivant les genres.

Groupes de discussion

\begin{tabular}{|l|l|l|l|l|}
\hline Dates & $\mathbf{2 8}$ avril 2011 & $\mathbf{2 8}$ octobre 2011 & $\mathbf{9}$ mars 2012 & $\mathbf{2 9}$ mars 2012 \\
\hline Participants & 7 immigrants & 9 Autochtones & 4 immigrants & 8 Autochtones \\
\hline Caractéristiques & $\begin{array}{l}\text { Étudiants + } \\
\text { professionnels }\end{array}$ & Étudiants & $\begin{array}{l}\text { Tous } \\
\text { professionnels }\end{array}$ & Tous étudiants \\
\hline
\end{tabular}

Toutes les discussions ont été enregistrées et retranscrites à la suite de chaque groupe de discussion. La recherche étant exploratoire, il nous a fallu faire ressortir les thèmes émergents suivant une perspective qui tienne compte du va-et-vient entre les différents contextes de vie et les expériences des participants. La démarche de la théorie enracinée 
(grounded theory) telle que suggérée par Strauss et Corbin (2004) nous a ainsi paru plus adaptée à nos analyses : les thèmes émergents ont été, dès que possible, nuancés afin de parvenir de façon inductive à des thèmes plus adaptés (Guillemette 2006; Paillé 1994). Parmi ceux-ci, quelques-uns nous ont semblé prédominants : l'incompréhension mutuelle, les stéréotypes, l'ignorance des vécus historiques des uns et des autres, le rôle quasi obnibulateur des discours publics, les rôles des organismes communautaires, la volonté de cheminer vers un dialogue.

\section{De l'incompréhension à la volonté de trouver des solutions}

Nos entrevues ont révélé - ce que nous soupçonnions au départ — qu'il existe beaucoup d'incompréhension de part et d'autre. Les nouveaux arrivants interviewés ont admis que l'image qu'ils avaient des Autochtones était plutôt négative. La grande majorité connaissait très peu de choses sur les communautés autochtones environnantes ou sur leur histoire. Pour la grande majorité des intervenants, leurs connaissances s'arrêtent principalement à ce qu'ils ont vu dans les films western avant leur arrivée au Canada (Groupe de discussion, 28 avril 2011). Une fois au Canada, la représentation qu'ils ont des Premières nations et des Métis se calque sur celle véhiculée par la société dominante. Ces derniers sont alors présentés comme des parias de la société. Selon un des participants, " on entend les gens dire que les Autochtones ne veulent pas travailler, qu'ils boivent trop et qu'ils reçoivent beaucoup d'argent du gouvernement. Si nous autres, nous ne sommes pas assez responsables pour connaître leur histoire, nous allons nous arrêter à ce que les autres disent d'eux » (Groupe de discussion, 28 avril 2011). Les participants reconnaissent toutefois que d'en savoir plus sur les questions autochtones permet une plus grande ouverture d'esprit :

[...] As Black people we are stigmatized in all sorts of ways, but nevertheless we the Black people still stigmatize Aboriginal people. When we think of Aboriginal people we see stereotypes, people picking up cans, a man asking for money. But that's not it. These people have suffered for centuries. When you talk and listen to them, and compare this to the stereotypes, it makes for two completely different worlds. (Groupe de discussion, 9 mars 2012)

Malgré les clichés, il existe une volonté d’outrepasser les stéréotypes même si ce désir paraît parfois insurmontable du fait du peu de contact social dans la sphère privée et professionnelle. L'individualisme ambiant ne favorise d'ailleurs pas les rapprochements selon les intervenants : "People here have learned to go to work, go home, watch tv. There is no human contact. I believe Aboriginal people do have this contact, as part of their traditions. But they have lost it here in the city» (Groupe de discussion, 9 mars 2012).

Les répondants sont conscients, jusqu'à un certain point, de faire partie du projet colonial canadien, mais ils ne se perçoivent pas comme colonisateur ou exécutant, mais bien comme surbordonnés à une structure coloniale déjà en place. Pour une des intervenantes, « cette obligation de s'intégrer à la société dominante classe les immigrants automatiquement dans le camp des adversaires pour les Autochtones car plus il y a d'immigrants au Canada, plus la 
population canadienne augmente et aussi les nouveaux arrivants apportent leurs cultures ceci réduit les avantages des Autochtones » (Groupe de discussion, 28 avril 2011). Certains vont même jusqu’à tracer un parallèle entre la colonisation des peuples autochtones canadiens et les colonisations des pays africains : "Les peuples africains [ont] aussi été victimes d'une discrimination raciale pendant la période coloniale. Les esclaves africains avaient été importés de l'Afrique vers l'Amérique où ils effectuaient des travaux forcés. Heureusement, les Africains ont récupéré leurs terres, mais les Autochtones canadiens, eux, n’ont pas pu récupérer leurs terres » (Groupe de discussion, 28 avril 2011). Cependant, les participants ne pensent pas avoir de responsabilités envers les traités qui ont été signés avec les peuples autochtones dans la mesure où ils ne les connaissent pas bien et qu'ils s'inscrivent dans le passé : «il faut qu’on ait une part pour pouvoir agir c'est-à-dire en politique. Si on n'est pas encore nous-mêmes bien assis comment est-ce qu'on peut commencer à prendre cette responsabilité à l'égard des autres » (Groupe de discussion, 28 avril 2011).

De leur côté, les intervenants autochtones connaissent peu de chose sur les nouveaux arrivants au Canada. Ce qu'ils connaissent de la politique d'immigration canadienne se limite à ce qu'ils ont entendu ou vu dans les médias. Toutefois, tous perçoivent la politique de multiculturalisme comme problématique :

what I know about immigration is in the context of racialized norms [...] So on the one-hand immigrants coming in who don't fit [the white anglo-norm] might be seen as less Canadian than say someone who is from Europe who is Anglo and white. Opposed to someone who is not Anglo, maybe someone from Africa, I don't know. So on the one hand immigrants [...] come here, they may experience some degree of racism, they don't fit this Anglo white norm of Canadianness. But on the other hand knowingly or not they are also benefiting from Indigenous dispossession, so in a way they are reproducing colonialism in Canada. (Groupe de discussion, 28 octobre 2011)

Ils pensent, de surcroit, que les immigrants ont une vision idyllique du Canada et que parfois le réveil est brutal. Ainsi, ce témoignage :

[In one of my course] there were four students from Iran, when they discovered that Canada had a residential school system they couldn't believe it. They had never heard of it, they couldn't believe that Canada would do something like that to Native people because Canada to them is this wonderful country, with freedom. Freedom in comparison to where they were from, they were shocked and amazed. That group of four wanted to discuss, where the students form Edmonton did not. I don't know how you convey that system, because history brings us to where we are now and I don't know how you tell this to new immigrants. (Groupe de discussion, 28 octobre 2011)

Même s'ils n'en sont pas toujours conscients, ces nouveaux arrivants ont, selon les Autochtones, une part de responsabilité par rapport aux traités, notamment le traité $n^{\circ} 6$, à Edmonton : "we are all Treaty Six people we are just on different sides of the agreement, and have rights and responsibilities to that agreement » (Groupe de discussion, 28 octobre 
2011). De ce fait même, les nouveaux arrivants se doivent d'apprendre l'histoire de ce pays pour comprendre la place des groupes autochtones dans cette histoire :

There is a greater responsibility for immigrants to learn the history of Indigenous people in Canada because it is where they are moving to [...] If this first step is done Indigenous people in this country would be more open to conversation and dialogue. We are all Treaty people and they need to understand that. (Groupe de discussion, 29 mars 2012)

Les participants, de part et d'autre, sont favorables au dialogue, mais certains obstacles paraissent insurmontables, entre autres à cause de la mauvaise presse dont font l'objet les deux groupes ${ }^{3}$. En outre, létablissement d'un dialogue n'est pas toujours dans les priorités des associations, des organismes et des institutions, souvent en mode survie ou en état de crise. Une chose est certaine, les Autochtones qui ont participé à l'étude pensent qu'il est temps qu'ils aient leur mot à dire en matière de politique d'immigration. Pour eux, le gouvernement fédéral est responsable en grande partie des barrières qui sérigent entre les peuples, quand traditionnellement il existait au Canada des processus et des cérémonies pour accueillir les nouveaux arrivants. C'est d'ailleurs, d'après eux, ces cérémonies et ces processus d'accueil autochtones qui ont permis au Canada d'exister.

\section{Conclusion}

La question de l'inclusion communautaire est une des plus discutées dans les débats autour de l'immigration au Canada. Généralement, ces discussions sont abordées à partir d'une double perspective (anglophone/francophone) qui paradoxalement exclut un des peuples fondateurs du Canada, les peuples autochtones. Pourtant, leurs traditions culturelles ont toujours véhiculé des éléments qui traduisent un esprit d'ouverture, d'accueil, nécessaire à ces pratiques d'inclusion tant recherchées dans la société canadienne actuelle. Il a été question, au cours de cette première phase de notre recherche, d'explorer à partir de deux perspectives périphériques (autochtone/africaine, francophone) si les possibilités d'un renouvellement du discours d'inclusion sont envisageables. Il nous a semblé de prime abord que si ces possibilités existent, si timides soient-elles, elles doivent faire l'impasse d'un discours de marginalisation qui a longtemps caractérisé l'histoire sociale canadienne. Ce discours est subreptice, mielleux. Il traverse tous les milieux communautaires, scolaires, etc., et sert encore de soubassement à plusieurs pratiques communautaires tant célébrées qui contribuent à fabriquer la société canadienne de demain. Pour les participants immigrants francophones, cet écueil se greffe parfois sur l'obstacle linguistique qui les éloigne davantage des milieux autochtones. Les participants à cette recherche ont reconnu la nécessité de déconstruire ces barrières afin de bâtir des ponts entre des populations qui, malgré certaines trajectoires similaires, semblent s'ignorer et ne pas encore savoir sur quels chemins se rencontrer; un pont qui serait peut-être un préalable au l'émergence de nouvelles valeurs qui serviront d'ancrage à ladite société inclusive et transculturelle.

3 « We only hear about immigrant people and Aboriginal people when something bad happens. So how can you empathize with each other if the picture you are getting of one another is always negative? » (Groupe de discussion, 29 mars 2012). 


\section{Bibliography}

\section{Sources primaires :}

Groupe de discussion, immigrants francophones, 28 avril 2011, Campus Saint-Jean, University of Alberta.

Groupe de discussion, autochtones, 28 octobre 2011, Faculty of Native Studies, University of Alberta.

Groupe de discussion, immigrants francophones, du 9 mars 2012, Cité francophone, Edmonton.

Groupe de discussion, autochtones, 29 mars 2012, Faculty of Native Studies, University of Alberta.

\section{Sources secondaires :}

Banting, K., et W. Kymlicka. 2010. "Canadian Multiculturalism: Global Anxieties and Local Debates ». British Journal of Canadian Studies, vol. 23 no 1: 43-72. http:// dx.doi.org/10.3828/bjcs.2010.3.

Campagne 2000. 2012. Nécessaire : un plan d'action fédéral pour éliminer la pauvreté des enfants et des familles au Canada. Toronto: Family Service Toronto. Consulté le 3 juin 2013. http://www.campaign2000.ca/2012ReportCardFr.pdf.

Chagnon, J. 2013. Statistique Canada. « Migrations internationales, 2010 et 2011 ». http:// www.statcan.gc.ca/pub/91-209-x/2013001/article/11787-fra.pdf.

Colin, Lindsay. 2007. Profils de communautés ethniques au Canada. La communauté

africaine au Canada, 2001. Ottawa : Ministre de l'Industrie. Consulté le 4 juin 2013. http:// www.statcan.gc.ca/pub/89-621-x/89-621-x2007010-eng.pdf.

Chui, T., Tran, K. et H. Maheux. 2007. Immigration au Canada. Un portrait de la population née à l'étranger, Recensement 2006. Ottawa : Ministre de l'Industrie. Consulté le 4 juin 2013. http://www12.statcan.ca/census-recensement/2006/as-sa/97-557/ pdf/97-557-XIF2006001.pdf.

Daniels, H. 1979. We are the New Nation: The Métis and National Native Policy. Ottawa: Native Council of Canada.

Davis, A. 2007. Femmes, race et classe. Paris : Antoinette Fougue, $2^{\mathrm{e}}$ Édition.

Dubar, C. 2000. La crise des identités : L'interprétation d'une mutation. Paris : PUF.

Denzin, N.K. et Y.S. Lincoln. 2005. The SAGE handbook of qualitative research.

Thousand Oaks: Sage Publications.

Farmer, D. et N. Labrie. 2008. «Immigration et francophonie dans les écoles ontariennes : comment se structurent les rapports entre les institutions, les parents et le monde communautaire? ». Revue des sciences de l'éducation, vol. 34, no 2 : 377-398.

Flanagan, T. 2000. First Nations? Second Thoughts, Montréal : McGill-Queen's University Press.

Gilmore, J. 2006. The immigrant labour force analysis series: The Canadian immigrant labour market in 2006: analysis by region or country of birth. Ottawa: Statistique Canada, Research Paper. 
Green, J. 2004. " Autodétermination, citoyenneté et fédéralisme : pour une relecture autochtone du palimpseste canadien ». Politique et sociétés, vol. 23, no 1 : 9-32.

Gouvernement du Canada. 2009. Comprendre la réalité « $3 \mathrm{M} »$ du Canada au XXI siècle. Rapport final, Juin 2009. Consulté le 3 juin 2013. http://www.policyresearch.gc.ca/ page.asp?pagenm=2009-0015_toc.

Guillemette, F. 2006. "Lapproche de la Grounded Theory; pour innover? ». Recherches Qualitatives, vol. $26: 32-50$.

Giust-Desprairies, F. et B. Müller. 1997. Se former dans un contexte de rencontres interculturelles. Paris : Anthropos.

Hage, G. 2001. " Polluting Memories: Migration and Colonial Responsibility in Australia » In 'Race' Panic and the Memory of Migration, ed. M. Morris and B. de Bary : 323-62. Hong Kong : Hong Kong University Press.

Labelle, M. et D. Salée. 1999. "La citoyenneté en question : l'État canadien face à l'immigration et la diversité nationale et culturelle ». Sociologie et sociétés, vol.31, no 2: 125-144.

Lawrence, B. et E. Dua. 2011. "Decolonizing Anti-Racism ». In Cultivating Canada: Reconciliation through the Lens of Cultural Diversity, ed. A. Mathur, J. Dewar et M. DeGagné : 235-257. Ottawa : Aboriginal Healing Foundation.

Lévesque, A. 2005. La santé des aînés francophones en milieu minoritaire - services, défis et obstacles. Winnipeg : Collège universitaire de Saint-Boniface.

Longhi, J. 2010. "La dimension sémantique du lien social dans les démocraties pluriculturelles ». In Vivre ensemble aujourd'hui. Le lien social dans les démocraties pluriculturelles, ed. E. Dacheux : 223-234. Paris : L'Harmattan.

Madariaga-Vignudo, L. 2009. Refugee-Aboriginal relations: A Case Study of a Canadian Inner City. M.A. Thesis. McGill University, Canada. Consulté le 4 juin 2013. http://digitool.library.mcgill.ca/R/?func=dbinjumpfull\&object_id=66680\&local_ base $=$ GEN01-MCG02.

Maingeneau, D. 1994, Lanalyse du discours : introduction aux lectures de l'archive. Paris : Hachette Université.

Newhouse, D. et E. Peters. 2003. «Aller de l'avant». In Des gens d'ici : Les autochtonesen milieu urbain, ed. D. Newhouse et E. Peters: 307-311. Ottawa : Projet de recherche sur les politiques (PRP).

Offord, Baden. 2008. "Landscapes of Exile (and Narratives on the Trauma of Belonging) ». In Landscapes of Exile: Once Perilous, Now Safe, ed. Anna Haebich et Baden Offord: 5-19. Bern: Peter Lang.

Paillé, P. 1994. "Lanalyse par théorisation ancrée ». Cahiers de recherche sociologique, vol $23: 147-181$.

Salée, D. 2005. «Peuples autochtones, racisme et pouvoir d'État en contextes canadien et québécois : Éléments pour une ré-analyse ». Nouvelles pratiques sociales, vol. 17, no $2: 54-74$.

Sanséau, P.-Y. 2005. "Les récits de vie comme stratégies d'accès au réel en sciences de gestion : Pertinence, positionnement et perspectives d'analyse ». Recherches qualitatives vol. 25 , no $2: 35-57$. 
Sébastien, H. 2006. "La notion d'identité personnelle en sociologie : analyse de la construction identitaire à partir du processus d'engagement ». Interrogations. Revue pluridisciplinaire en sciences de l'homme et de la société, no 3 : 126-134.

Snoeckx, M. 2000. "Construction des identités professionnelles en formation initiale: approche expérientielle et direction de mémoires ». In Enseignants formateurs : La construction de l'identité professionnelle, ed. C. Gohier et C. Alin : 213-237. Paris : L'Harmattan.

Statistique Canada. 2013a. «Enquête nationale auprès des ménages de 2011: Les peuples

autochtones au Canada : Premières Nations, Métis et Inuits ». Le Quotidien, mercredi 8 mai. Consulté le 3 juin 2013. http://www12.statcan.ca/census-recensement/2006/ as-sa/97-558/p1-fra.cfm.

Statistique Canada. 2013b. «Enquête nationale auprès des ménages de 2011 : Immigration, lieu de naissance, citoyenneté, origine ethnique, minorités visibles, langue et religion ». Le Quotidien, mercredi 8 mai. Consulté le 3 juin 2013. http://www. statcan.gc.ca/daily-quotidien/130508/dq130508-fra.pdf.

Statistique Canada. 2013c. Profil de l'enquête nationale auprès des ménages, Edmonton RMR, Alberta, 2011. Consulté le $1^{\text {er }}$ mars 2013. http://www12.statcan.gc.ca/nhsenm/2011/dp-pd/prof/index.cfm?Lang=F.

Statistique Canada, 2006. «Portrait des communautés de langue officielle au Canada : un outil d'analyse utile ». Le Quotidien, mardi 3 mars. Consulté le 4 juin 2013.

Strauss, A., et J. Corbin. 2004. Les fondements de la recherche qualitative: techniques et procédures de développement de la théorie enracinée. Fribourg : Academic Press.

Thobani, S. 2007. Exalted Subjects: Studies in the Making of Race and Nation in Canada. Toronto: University of Toronto Press.

Thurston,W, A. McGrath et K. Sehgal. 1993. With two pennies in my pocket: I feel not so helpless. A Report on the Mental and Occupational Health Promotion Needs of Immigrants in Calgary. Calgary: Canadian Mental Health Association.

Ville de Vancouver. 2011. Vancouver Dialogues: First Nations, Urban Aboriginal and Immigrant Communities. Vancouver: Social Policy, City of Vancouver. 